Retraction

\title{
Retracted: VEGF Genetic Polymorphisms May Contribute to the Risk of Diabetic Nephropathy in Patients with Diabetes Mellitus: A Meta-Analysis
}

\author{
The Scientific World Journal
}

Received 19 May 2020; Accepted 26 June 2020; Published 1 August 2020

Copyright (c) 2020 The Scientific World Journal. This is an open access article distributed under the Creative Commons Attribution License, which permits unrestricted use, distribution, and reproduction in any medium, provided the original work is properly cited.

The Scientific World Journal has retracted the article titled "VEGF Genetic Polymorphisms May Contribute to the Risk of Diabetic Nephropathy in Patients with Diabetes Mellitus: A Meta-Analysis" [1]. This article is one of a series of very similar meta-analyses written by different authors that were published in 2014 and 2015, characterized by searching the complementary and alternative medicine database CISCOM despite the topic not being about CAM [2], as is also the case with this article. The overlaps of wording with these articles are concentrated in the Materials and Methods and Results sections, and a paragraph in the Discussion. The article also mentions "Begger's test," which is a mistaken combination of "Begg's test" and "Egger's test" and is also characteristic of this series of articles. The article inappropriately mentions other cancers and genes, including gastric cancer in the abstract and "breast cancer" and "IFN- $\gamma$ gene" in Figure 1 in the eligibility exclusion box, whereas the article is about diabetic nephropathy (DN) and VEGF.

Additionally, the assessment of publication bias may be incorrect. The statement that "Egger's test also did not display strong statistical evidence for publication bias (allele mode: $t=2.92, P=0.011$ and dominant model: $t=2.53$, $P=0.024$, resp.)" is made despite the test result being statistically significant and the funnel plots are said to be symmetrical, though the smaller studies (with greater standard error) show greater associations with $\mathrm{DN}$, which is an indication of publication bias.

The authors said they attended a course on writing metaanalyses that lead to incorrect data analysis and they apologised for the mistakes. However, they asked to retract the article (though they did not approve the content of this notice) and this was approved by the editorial board.

\section{References}

[1] L. Sun, Q. Yuan, N. Cao et al., "VEGF Genetic Polymorphisms May Contribute to the Risk of Diabetic Nephropathy in Patients with Diabetes Mellitus: A Meta-Analysis," The Scientific World Journal, vol. 2014, Article ID 624573, 11 pages, 2014.

[2] G. Filion, "A Flurry of Copycats on PubMed," The Grand Locus, 2014, http://blog.thegrandlocus.com/2014/10/a-flurryof-copycats-on-pubmed. 


\title{
VEGF Genetic Polymorphisms May Contribute to the Risk of Diabetic Nephropathy in Patients with Diabetes Mellitus: A Meta-Analysis
}

\author{
Li Sun, ${ }^{1}$ Quan Yuan, ${ }^{2}$ Ning Cao, ${ }^{3}$ Wei Guo, ${ }^{4}$ Li Yao, ${ }^{1}$ Jiang-Min Feng, \\ Jian-Fei Ma, ${ }^{1}$ and Li-Ning Wang ${ }^{1}$ \\ ${ }^{1}$ Department of Nephrology, The First Affiliated Hospital of China Medical University, Shenyang 110001, China \\ ${ }^{2}$ Department of Orthopedics, Shengjing Hospital of China Medical University, Shenyang 110004, China \\ ${ }^{3}$ Department of Blood Purification, General Hospital of Shenyang Military Region, Shenyang 110015, China \\ ${ }^{4}$ Department of Nephrology, The Fourth People's Hospital of Shenyang, Shenyang 110034, China
}

Correspondence should be addressed to Li Sun; cmulh_sl@126.com

Received 8 May 2014; Accepted 6 July 2014; Published 11 August 2014

Academic Editor: Gotthard Kunze

Copyright (C $2014 \mathrm{Li}$ Sun et al. This is an open access article distributed under the Creative Commons Attribution License, which permits unrestricted use, distribution, and reproduction in any medium, provided the original work is properly cited.

Objective. This meta-analysis aimed to investigate a comprehensive and reliable conclusion on the correlations of single nucleotide polymorphisms (SNPs) in the vascular endothelial growth factor (VEGF) gene with the risk of diabetic nephropathy (DN) in patients with diabetes mellitus (DM). Methods. We screened PubMed, Embase, Web of Science, Cochrane Library, CISCOM, CINAHL, Google Scholar, CBM, and CNKI databases for those relevant studies that investigated the association of 14,945 subjects with clinicopathological parameters in gastric cancer. Results. Eleven case-control studies that met all inclusion criteria were included in this meta-analysis. A total of 14,945 subjects were involved, including 3,049 DN patients and 11,896 DM patients. Our meta-analysis results revealed that VEGF rs2010963 and rs3025039 polymorphisms might contribute to the risk of DN in DM patients. Ethnicity-stratified analysis suggested that VEGF genetic polymorphisms were associated with an increased risk of DN among Asians. However, we found no correlations of VEGF genetic polymorphisms with susceptibility to DN among Caucasians. Conclusion. Our findings suggest that VEGF rs2010963 and rs3025039 polymorphisms may contribute to the risk of DN in DM patients, especially among Asians. Thus, VEGF genetic polymorphisms could be useful biomarkers for early diagnosis of DN in DM patients.

\section{Introduction}

Diabetic nephropathy (DN), also identical to intercapillary glomerulonephritis or nodular diabetic glomerulosclerosis, is among the most lethal complications that occur in patients with type 1 or type 2 diabetes mellitus (DM) [1, 2]. It is well known that DN can cause death two or three years after the initial lesions appear; it is more frequent in men and has become a secondary cause of death among endstage renal diseases [3-5]. In general, DN is a multifactorial disease induced by complex interactions between environmental factors and genetic determinants $[6,7]$. Several factors always contributing to DN risk include abnormal renal hemodynamic responses, fatty acid metabolism caused by hyperglycemia, hypertension, and abnormal metabolism of vasoactive substances [8]. Currently, vascular endothelial growth factor (VEGF) has also been reported to be associated with the development and progression of DN $[9,10]$.

$V E G F$ is a secreted mitogen highly specific for vascular endothelial cells, which have been implicated in endothelial cell proliferation and migration [11]. The human VEGF gene is located on chromosome 6p21.3 and composed of 8 exons and 7 introns, with an overall length of approximately $14 \mathrm{~kb}$ [12]. As a potent multifunctional cytokine, VEGF has been 
commonly considered as a prime determinant and regulator of angiogenesis, vasculogenesis, and vascular permeability $[13,14]$. A number of epidemiological studies have demonstrated that VEGF plays a crucial role in the pathogenesis of diabetic microvascular complications, including diabetic retinopathy and DN [15-17]. VEGF is responsible for mediating angiogenesis by increasing vascular permeability to water and proteins, while excessive vascular permeability during pathological angiogenesis may contribute to the development and progression of DN [18]. In addition, genetic variations in the VEGF gene might lead to high-level expression of VEGF. However, high expression of VEGF may alter intracellular signal transduction, promote extracellular matrix synthesis, and stimulate renal hypertrophy, which are thought to be key factors in the increase of susceptibility to DN $[19,20]$. Therefore, it was postulated that single nucleotide polymorphisms (SNPs) in the VEGF gene could be functional and were associated with an increased risk of DN [21]. Abundant studies have indicated that VEGF genetic polymorphisms may play an important role in the development of DN [22, 23]. However, results of these studies have been contradictory [24-26]. This meta-analysis was performed to evaluate the relationships of VEGF genetic polymorphisms with the risk of $\mathrm{DN}$ in DM patients.

\section{Materials and Methods}

2.1. Literature Search. Studies addressing the correlations of VEGF genetic polymorphisms with the risk of diabetic nephropathy in patients with diabetes mellitus were identified by searching for articles in the following electronic databases: the Medline (1966 2013), the Cochrane Library Database (Issue 12, 2013), Embase (1980 2013), PubMed (1966 2013), CINAHL (1982 2013), Web of Science (1945 2013), and Chinese Biomedical Database (CBM) (1982 2013). Various combination of keywords and MeSH terms: "SNP" or "mutation" or "genetic polymorphism" or "variation" or "polymorphism" or "single nucleotide polymorphism" or "variant"] and ["diabetic nephropathies" or "DN" or "diabetic kidney disease"] and ["vascular endothelial growth factors" or "VEGF" or "vascular permeability factor" or "VPF"]. We also performed a manual search of the reference lists from the relevant articles to find other potential articles.

2.2. Selection Criteria. The eligible studies included in our meta-analysis should meet the following types of inclusion criteria: (1) the study design must be clinical cohort or casecontrol study; (2) the study must relate to the relationships of VEGF genetic polymorphisms with the risk of DN in DM patients; (3) all patients must conform to the diagnostic criteria of DN; (4) the study must provide sufficient information about the frequencies of SNPs. If the study could not meet the inclusion criteria, it would be excluded. The most recent or the largest sample size publication was included when the authors published several studies using the same subjects.

2.3. Data Extraction. Two authors from each included study used a standardized form to systematically collect relevant data. The researchers collected the following data: the first author and publication year of article, language of publication, geographical location, design of study, sample size, the source of the subjects, allele frequencies, source of samples, genotyping method of SNP, evidence of Hardy-Weinberg equilibrium (HWE), and so forth.

2.4. Quality Assessment. Methodological quality was independently assessed by two researchers according to the Newcastle-Ottawa Scale (NOS) criteria [27]. The NOS criteria included three aspects: (1) subject selection: 0 4; (2) comparability of subject: $0 \sim 2$; (3) clinical outcome: $0 \sim 3$. NOS scores ranged from 0 to 9 , and a score of $\geq 7$ indicates a good quality.

2.5. Statistical Analysis. The STATA version 12.0 (Stata Corp, College Station, TX, USA) software was used for metaanalysis. We calculated crude odds ratios (ORs) with their 95\% confidence intervals $(95 \% \mathrm{CI})$ to evaluate their relationships under 5 genetic models: the allele model (mutant [M] allele versus wild [W] allele), the dominant model ( $\mathrm{WM}+\mathrm{MM}$ versus $\mathrm{WW}$ ), the recessive model (MM versus $\mathrm{WW}+\mathrm{WM}$ ), the homozygous model (MM versus $\mathrm{WW}$ ), and the heterozygous model (MM versus WM). The $Z$ test was used to estimate the statistical significance of pooled statistics. The Cochran's Q-statistic and $I^{2}$ test were used to evaluate potential heterogeneity between studies [28]. If Q-test shows a $P<0.05$ or $I^{2}$ test exhibits $>50 \%$ which indicates significant heterogeneity, the random effect model was conducted or else the fixed effects model was used. We also performed subgroup and metaregression analyses to investigate potential sources of heterogeneity. In order to evaluate the influence of single studies on the overall estimate, sensitivity analysis was performed. We conducted Begger's funnel plots and Egger's linear regression test to investigate publication bias [29].

\section{Results}

3.1. Characteristics of Included Studies. Initially, the searched keywords identified 308 articles. We reviewed the titles and abstracts of all articles and excluded 154 articles; full texts and data integrity were also reviewed and 142 articles were further excluded (Figure 1). Finally, 11 case-control studies were included in this meta-analysis [18, 22-26, 30-34]. Publication years of the eligible studies ranged from 2005 to 2013. The distribution of the number of topic-related literatures in electronic databases during the last decade is shown in Figure 2. A total of 14,945 subjects were involved, including 3,049 DN patients and 11,896 DM patients. Overall, eight studies were conducted among Asians, and three studies were conducted among Caucasians. The classical polymerase chain reaction-restriction fragment length polymorphism (PCR-RELP) method was performed in eight studies, while the other three studies used TaqMan assay, PCR-amplicon sequence analysis (PCR-ASA), and Pyrosequencing methods. The NOS scores of all included studies were $\geq 5$. We summarized the study characteristics and methodological quality in Table 1. 


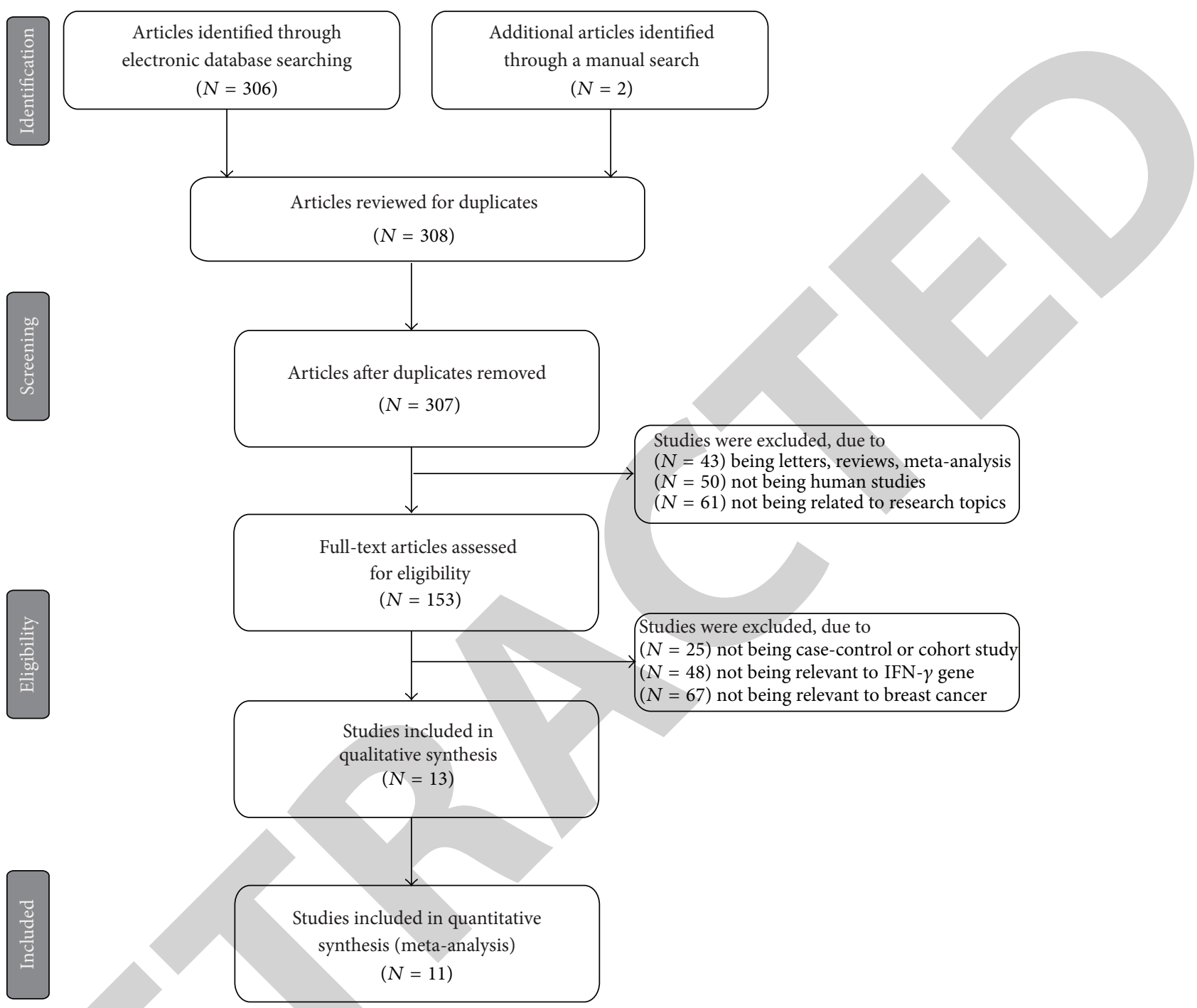

FIGURE 1: Flow chart of literature search and study selection. Eleven case-control studies were included in this meta-analysis.

3.2. Quantitative Data Synthesis. Meta-analysis findings on the relationships of VEGF genetic polymorphisms with the risk of DN in DM patients were shown in Table 2. The random effects model was conducted since significant heterogeneity existed between studies. Our meta-analysis results revealed that VEGF rs2010963 (allele model: $\mathrm{OR}=1.26,95 \% \mathrm{CI}=$ 1.05 1.52, $P=0.015$; dominant model: $\mathrm{OR}=1.29,95 \% \mathrm{CI}=$ 1.05 1.59, $P=0.015$; recessive model: $\mathrm{OR}=1.44,95 \% \mathrm{CI}=$ 1.00 2.05, $P=0.047$; homozygous model: $\mathrm{OR}=1.65,95 \%$ $\mathrm{CI}=1.08 \sim 2.50, P=0.020$; resp.) and rs3025039 (allele model: $\mathrm{OR}=1.38,95 \% \mathrm{CI}=1.06 \sim 1.80, P=0.018$; dominant model: $\mathrm{OR}=1.53,95 \% \mathrm{CI}=1.02 \sim 2.27, P=0.037$; homozygous model: $\mathrm{OR}=1.56,95 \% \mathrm{CI}=1.06 \sim 2.30, P=0.023$; resp.) polymorphisms might contribute to the risk of $\mathrm{DN}$ in DM patients (Figure 3). Nevertheless, no similar associations were found in other polymorphisms in the VEGF gene (all $P>$ 0.05).

Subgroup analysis by ethnicity suggested that VEGF genetic polymorphisms were associated with an increased risk of DN among Asians (allele model: $\mathrm{OR}=1.36,95 \% \mathrm{CI}=$ 1.17 1.59, $P<0.001$; dominant model: $\mathrm{OR}=1.46,95 \% \mathrm{CI}=$ 1.19 1.79, $P<0.001$; recessive model: $\mathrm{OR}=1.46,95 \% \mathrm{CI}=$ 1.12 1.91, $P=0.005$; homozygous model: $\mathrm{OR}=1.72,95 \% \mathrm{CI}=$ 1.27 2.31, $P<0.001$; resp.) (Figure 4). However, we found no correlations of VEGF genetic polymorphisms with susceptibility to DN among Caucasians (all $P>0.05$ ). Further subgroup analysis by genotyping method showed significant associations between VEGF genetic polymorphisms and DN risk in the PCR-RFLP subgroup (allele model: OR $=1.31,95 \%$ $\mathrm{CI}=1.13 \sim 1.52, P<0.001 ;$ dominant model: $\mathrm{OR}=1.44,95 \%$ $\mathrm{CI}=1.21 \sim 1.71, P<0.001 ;$ recessive model: $\mathrm{OR}=1.32,95 \%$ $\mathrm{CI}=1.03 \sim 1.70, P=0.029$; homozygous model: $\mathrm{OR}=1.57$, 95\% $\mathrm{CI}=1.18 \sim 2.10, P=0.002$; resp.) but not in the non-PCRRFLP subgroup (all $P>0.05$ ).

Metaregression analysis confirmed that SNP type and ethnicity might be the main sources of heterogeneity (as shown in Table 3). The results of sensitivity analysis suggested that no single study could influence the overall pooled 


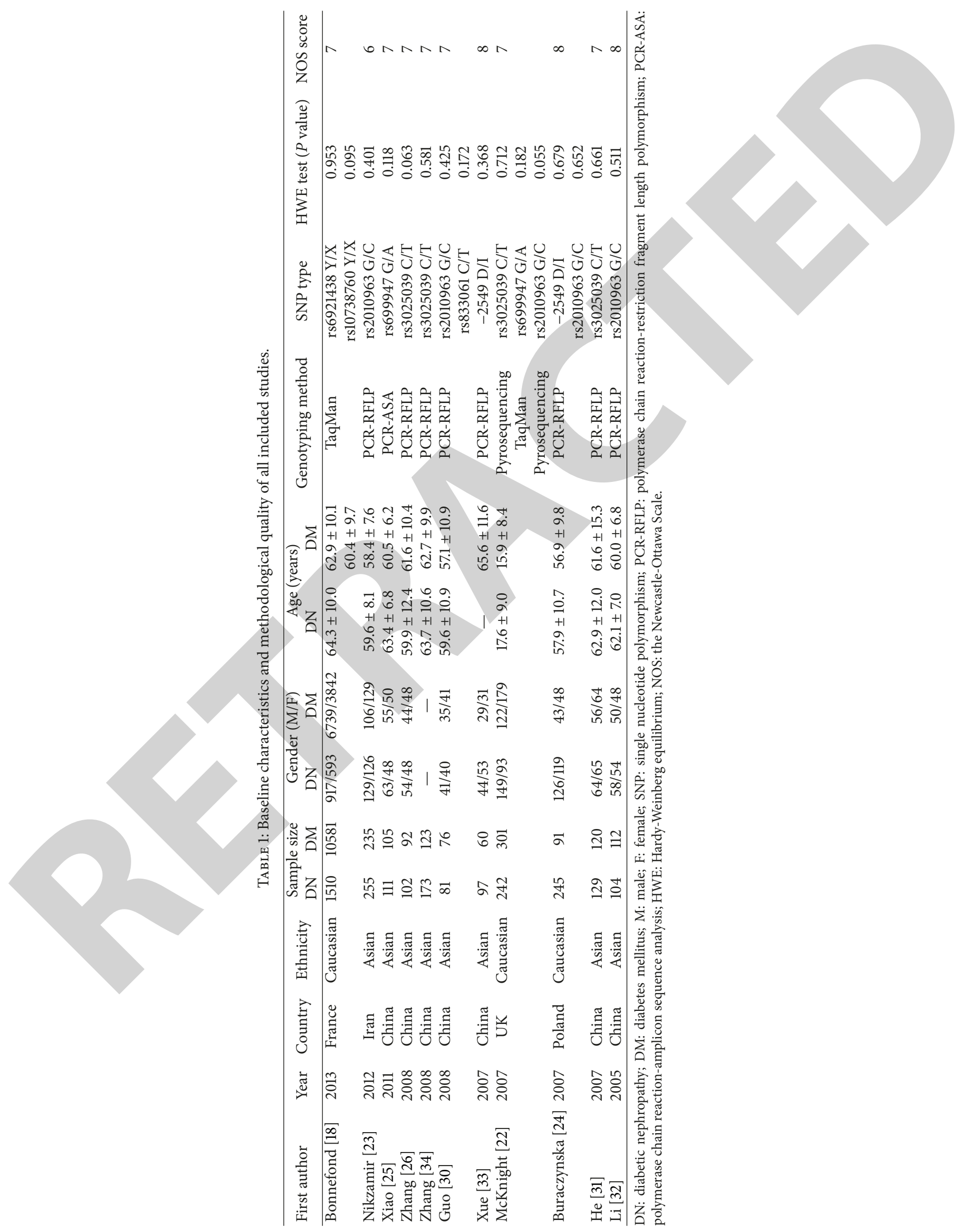


TABLE 2: Meta-analysis of the relationships of VEGF genetic polymorphisms with the risk of diabetic nephropathy in patients with diabetes mellitus.

\begin{tabular}{|c|c|c|c|c|c|c|c|c|c|c|c|c|c|c|c|}
\hline & \multicolumn{3}{|c|}{$\begin{array}{l}\text { M allele vs. W allele } \\
\text { (Allele model) }\end{array}$} & \multicolumn{3}{|c|}{$\begin{array}{l}\text { WM + MM vs. WW } \\
\text { (Dominant model) }\end{array}$} & \multicolumn{3}{|c|}{$\begin{array}{l}\text { MM vs. WW + WM } \\
\text { (Recessive model) }\end{array}$} & \multicolumn{3}{|c|}{$\begin{array}{c}\text { MM vs. WW } \\
\text { (Homozygous model) }\end{array}$} & \multicolumn{3}{|c|}{$\begin{array}{c}\text { MM vs. WM } \\
\text { (Heterozygous model) }\end{array}$} \\
\hline & OR & $95 \%$ CI & $P$ & OR & $95 \%$ CI & $P$ & OR & $95 \%$ CI & $P$ & OR & $95 \%$ CI & $P$ & OR & $95 \% \mathrm{CI}$ & $P$ \\
\hline 11 & 1.17 & $1.05-1.29$ & 0.004 & 1.20 & $1.04-1.39$ & 0.010 & 1.17 & $1.02-1.34$ & 0.030 & 1.29 & $1.06-1.56$ & 0.010 & 1.12 & $0.98-1.28$ & 0.103 \\
\hline \multicolumn{16}{|c|}{ SNP } \\
\hline rs6921438 Y/X & 0.88 & $0.76-1.04$ & 0.130 & 0.83 & $0.65-1.06$ & 0.129 & 0.88 & $0.67-1.16$ & 0.366 & 0.79 & $0.58-1.09$ & 0.152 & 0.94 & -1.26 & 0.682 \\
\hline rs10738760 Y/X & 1.08 & $0.93-1.27$ & 0.316 & 1.13 & $0.88-1.45$ & 0.339 & 1.09 & $0.84-1.41$ & 0.522 & 1.16 & $0.85-1.58$ & 0.337 & 1.05 & $0.79-1.38$ & 0.751 \\
\hline rs2010963 G/C & 1.26 & $1.05-1.52$ & 0.015 & 1.29 & $1.05-1.59$ & 0.015 & 1.44 & $1.00-2.05$ & 0.047 & 1.65 & $1.08-2.50$ & 0.020 & 1.28 & $0.94-1.74$ & 0.115 \\
\hline rs699947 G/A & 1.04 & $0.85-1.29$ & 0.668 & 0.95 & $0.69-1.30$ & 0.736 & 1.22 & $0.85-1.75$ & 0.288 & 1.15 & $0.75-1.76$ & 0.516 & 1.29 & $0.86-1.96$ & 0.223 \\
\hline $\mathrm{rs} 3025039 \mathrm{C} / \mathrm{T}$ & 1.38 & $1.06-1.80$ & 0.018 & 1.53 & $1.02-2.27$ & 0.037 & 1.39 & $0.98-1.98$ & 0.062 & 1.56 & $1.06-2.30$ & 0.023 & 1.27 & $0.87-1.84$ & 0.210 \\
\hline rs833061 C/T & 1.17 & $0.74-1.83$ & 0.502 & 1.16 & $0.59-2.29$ & 0.659 & 1.42 & $0.57-3.55$ & 0.449 & 1.50 & $0.54-4.16$ & 0.431 & 1.38 & $0.53-3.56$ & 0.507 \\
\hline $2549 \mathrm{D} / \mathrm{I}$ & 1.12 & $0.84-1.50$ & 0.442 & 1.27 & $0.84-1.92$ & 0.251 & 0.98 & $0.57-1.69$ & 0.951 & 1.13 & $0.62-2.08$ & 0.684 & 0.88 & $0.50-1.56$ & 0.661 \\
\hline \multicolumn{16}{|c|}{ Ethnicity } \\
\hline Caucasians & 1.03 & $0.95-1.12$ & 0.449 & 1.02 & $0.90-1.16$ & 0.711 & 1.07 & $0.93-1.23$ & 0.368 & 1.07 & $0.91-1.26$ & 0.421 & 1.07 & $0.92-1.24$ & 0.401 \\
\hline Asians & 1.36 & $1.17-1.59$ & $<0.001$ & 1.46 & $1.19-1.79$ & $<0.001$ & 1.46 & $1.12-1.91$ & 0.005 & 1.72 & $1.27-2.31$ & $<0.001$ & 1.31 & $0.99-1.74$ & 0.061 \\
\hline \multicolumn{16}{|c|}{ Method } \\
\hline Non-PCR-PFLP & 1.04 & $0.94-1.14$ & 0.453 & 1.00 & $0.88-1.15$ & 0.944 & 1.10 & $0.94-1.28$ & 0.247 & 1.11 & $0.90-1.36$ & 0.344 & 1.10 & $0.94-1.29$ & 0.232 \\
\hline PCR-RFLP & 1.31 & $1.13-1.52$ & $<0.001$ & 1.44 & $1.21-1.71$ & $<0.001$ & 1.32 & $1.03-1.70$ & 0.029 & 1.57 & $1.18-2.10$ & 0.002 & 1.16 & $0.90-1.51$ & 0.243 \\
\hline
\end{tabular}

W: wild allele; M: mutant allele; WW: wild homozygote; WM: heterozygote; MM: mutant homozygote; OR: odds ratio; 95\%CI: 95\% confidence interval; SNP: single nucleotide polymorphism.

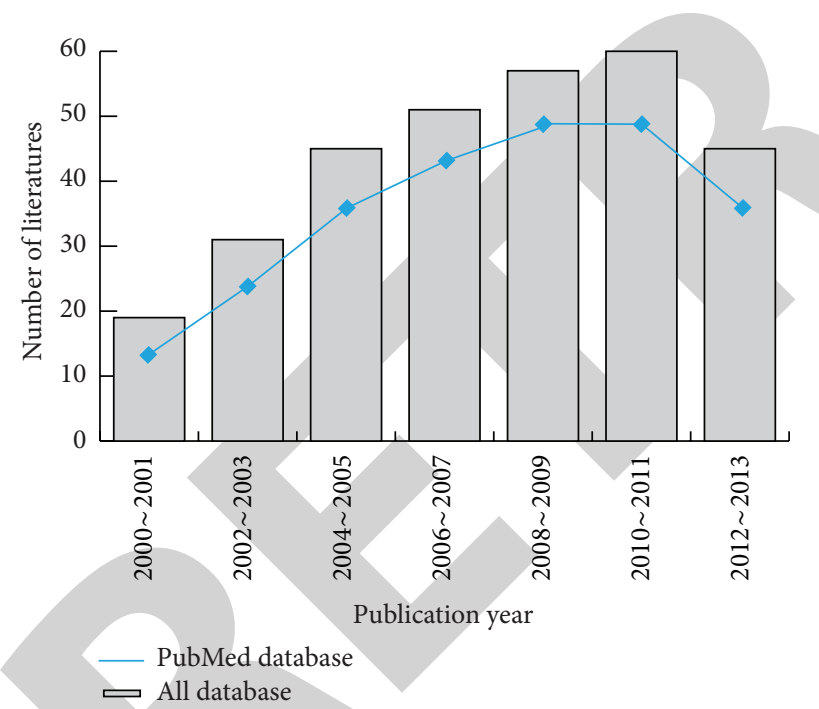

FIGURE 2: Distribution of the number of topic-related literatures in the electronic database during the last decade.

estimates (Figure 5). We found no evidence of obvious asymmetry in the Begger's funnel plots (Figure 6). Egger's test also did not display strong statistical evidence for publication bias (allele mode: $t=2.92, P=0.011$ and dominant model: $t=2.53, P=0.024$, resp.).

\section{Discussion}

VEGF is a potent multifunctional cytokine belonging to the multipotent cytokines family [35]. The role of VEGF has
TABLE 3: Univariate and multivariate metaregression analyses of potential source of heterogeneity.

\begin{tabular}{|c|c|c|c|c|c|c|}
\hline \multirow{2}{*}{$\begin{array}{l}\text { Heterogeneity } \\
\text { factors }\end{array}$} & \multirow{2}{*}{ Coefficient } & \multirow{2}{*}{ SE } & \multirow{2}{*}{$Z$} & \multirow{2}{*}{$P$} & \multicolumn{2}{|c|}{$95 \% \mathrm{CI}$} \\
\hline & & & & & LL & UL \\
\hline \multicolumn{7}{|l|}{ Publication year } \\
\hline Univariate & -0.349 & 0.016 & -2.21 & 0.027 & -0.066 & -0.004 \\
\hline Multivariate & -0.062 & 0.035 & -1.75 & 0.080 & -0.132 & 0.007 \\
\hline \multicolumn{7}{|l|}{ SNP type } \\
\hline Univariate & -0.063 & 0.026 & -2.43 & 0.015 & -0.113 & -0.012 \\
\hline Multivariate & -0.037 & 0.033 & -1.13 & 0.258 & -0.101 & 0.027 \\
\hline \multicolumn{7}{|l|}{ Country } \\
\hline Univariate & 0.031 & 0.040 & 0.79 & 0.431 & -0.046 & 0.108 \\
\hline Multivariate & -0.101 & 0.080 & -1.26 & 0.206 & -0.257 & 0.055 \\
\hline \multicolumn{7}{|l|}{ Ethnicity } \\
\hline Univariate & 0.264 & 0.088 & 2.99 & 0.003 & 0.091 & 0.437 \\
\hline Multivariate & 0.199 & 0.088 & 2.25 & 0.025 & 0.025 & 0.372 \\
\hline \multicolumn{7}{|l|}{ Method } \\
\hline Univariate & -0.222 & 0.088 & -2.52 & 0.012 & -0.395 & -0.049 \\
\hline Multivariate & -0.047 & 0.137 & -0.34 & 0.733 & -0.315 & 0.222 \\
\hline
\end{tabular}

SE: standard error; 95\%CI: 95\% confidence interval; UL: upper limit; LL: lower limit; SNP: single nucleotide polymorphism.

been primarily investigated in its regulation of angiogenesis $[9,18]$. It should be noted that VEGF mediates angiogenesis in part by increasing normal vascular permeability to water and proteins [9]. However, excessive vascular permeability during pathological angiogenesis may in turn conduce to macroor microvascular diseases in some way [18]. Recently, high VEGF concentrations have been detected in patients with 
Allele model (M allele versus W allele)

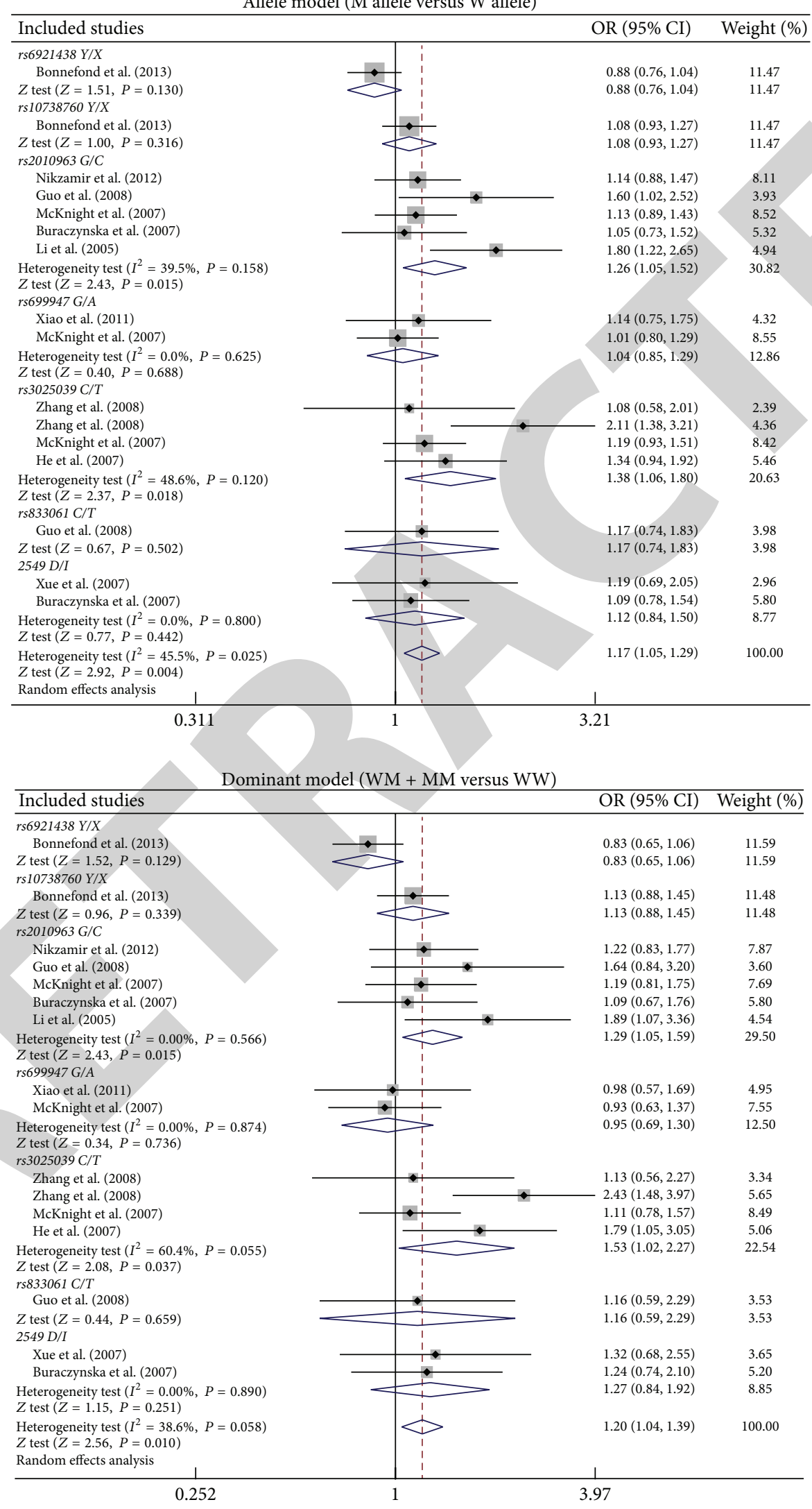

FIGURE 3: Forest plots for the relationships of VEGF genetic polymorphisms with the risk of diabetic nephropathy in patients with diabetes mellitus under the allele and dominant models. 
Allele model ( $\mathrm{M}$ allele versus $\mathrm{W}$ allele)

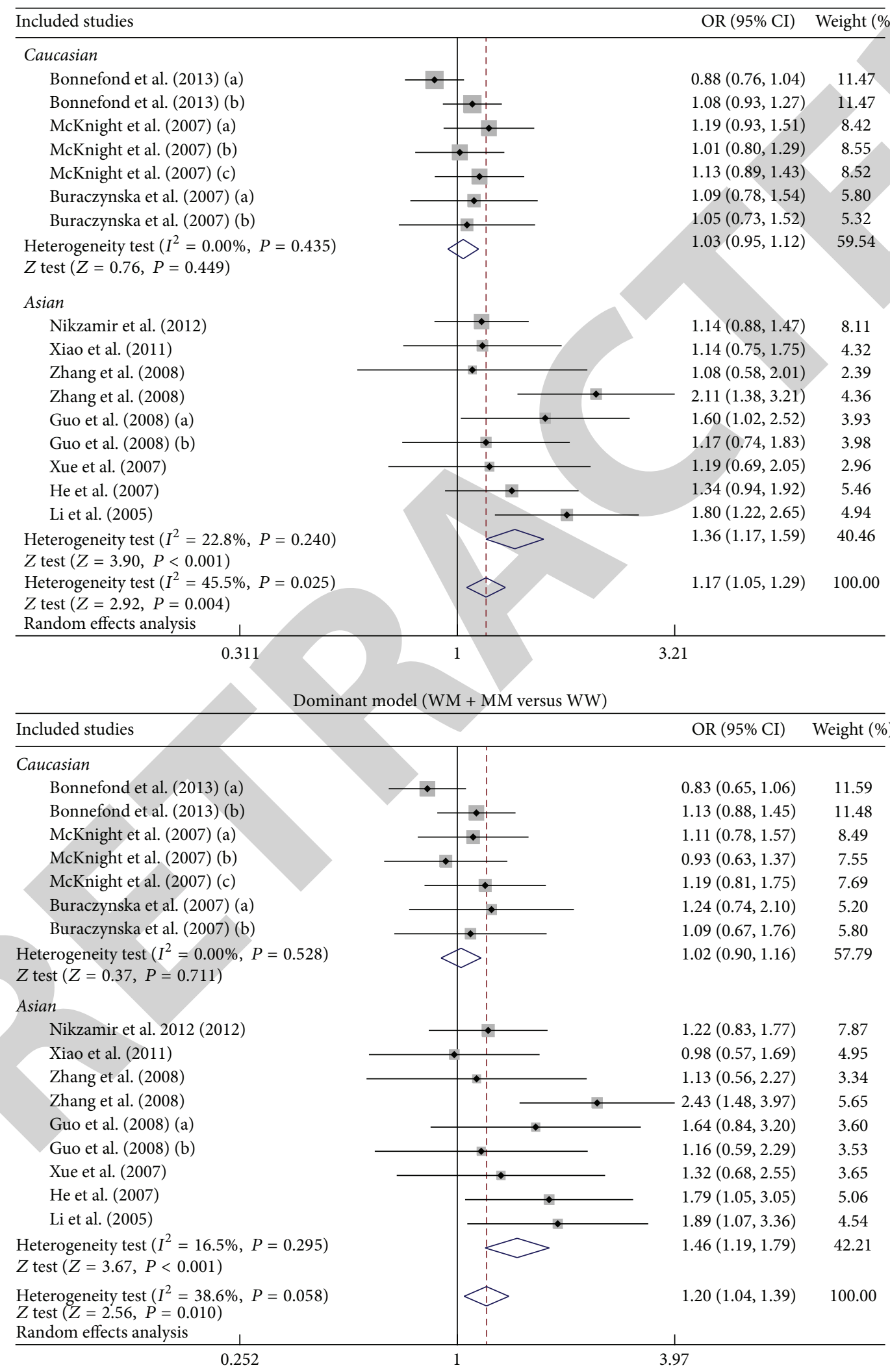

FIGURE 4: Subgroup analyses by ethnicity of the relationships of VEGF genetic polymorphisms with the risk of diabetic nephropathy in patients with diabetes mellitus under the allele and dominant models. 


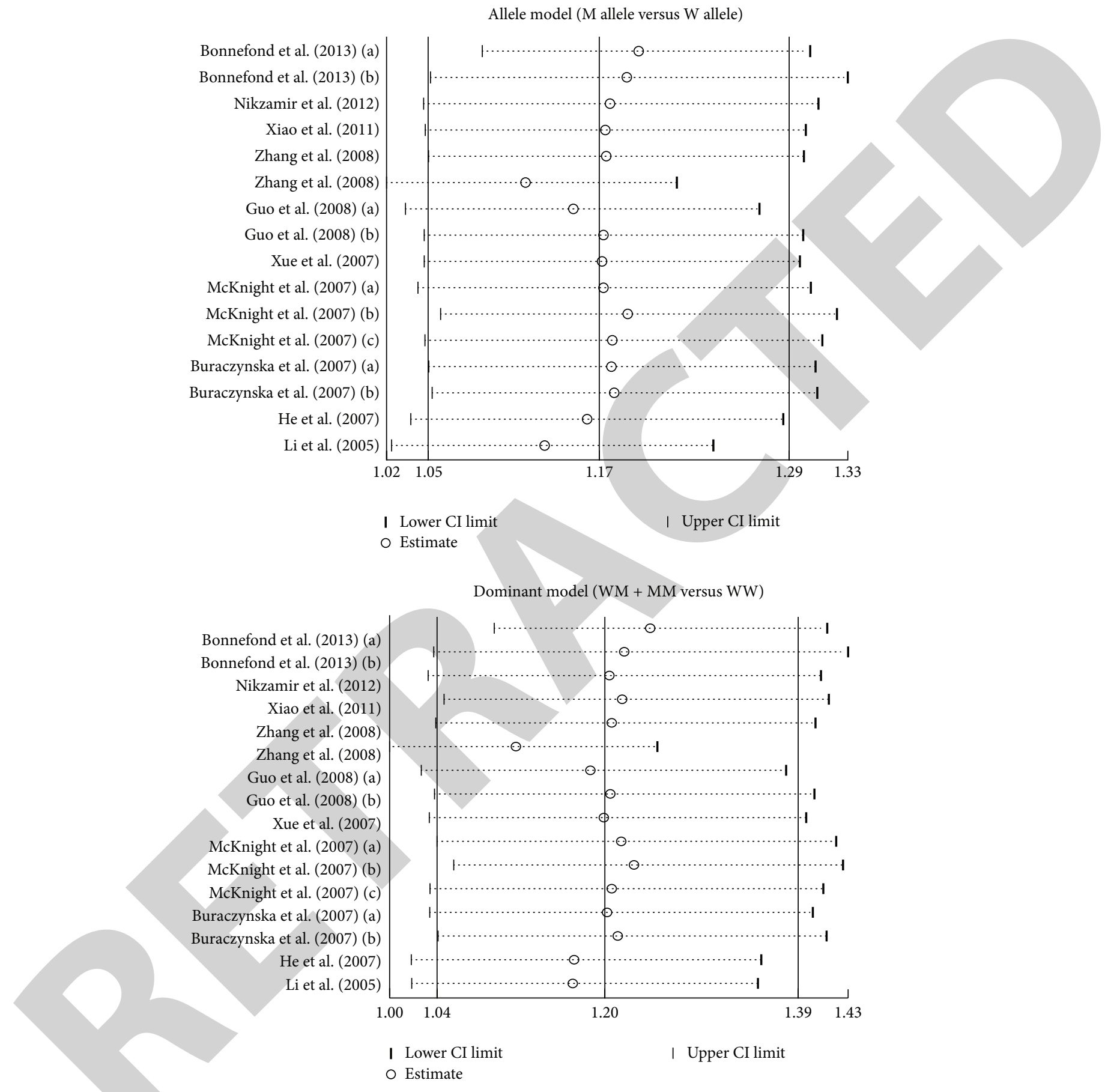

FIGURE 5: Sensitivity analysis of the summary odds ratio coefficients on the relationships of VEGF genetic polymorphisms with the risk of diabetic nephropathy in patients with diabetes mellitus under the allele and dominant models.

respiratory diseases or diabetic microvascular complications including DN, which indicated VEGF might play a major role in the susceptibility to DN $[18,36]$. It is widely accepted that VEGF was involved in the induction and maintenance of the fenestrae in glomerular capillary endothelial cells [37, 38], while genetic abnormalities in VEGF gene may result in the aberrancy of glomerular filtration barrier and renal tubular reabsorption, which can eventually lead to the occurrence of microalbuminuria and proteinuria $[39,40]$. Therefore, $V E G F$ genetic variants have been postulated to be clearly an excellent candidate for the pathogenesis of DN [21, 41].

In the present meta-analysis, we evaluated the relationships of VEGF genetic polymorphisms with the risk of DN in DM patients. Finally, 11 independent case-control studies were included. Our meta-analysis results showed positive associations of VEGF rs2010963 and rs3025039 

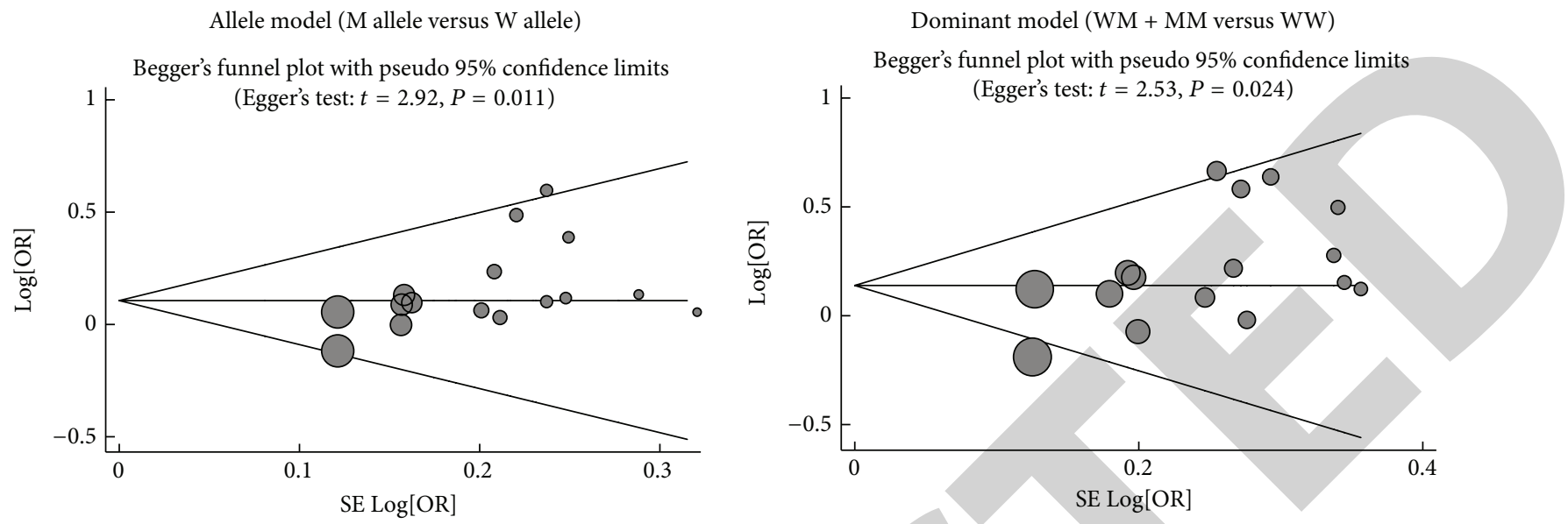

FIGURE 6: Begger's funnel plot of publication biases on the relationships of VEGF genetic polymorphisms with the risk of diabetic nephropathy in patients with diabetes mellitus under the allele and dominant models.

polymorphisms with susceptibility to DN in DM patients, indicating that these polymorphisms may be causative factors for the incidence of DN. Although the exact function of VEGF gene in the development of DN is not fully understood, a potential explanation might be that variants in the VEGF gene increased its expression level which was suspected to play a vital role in injury of podocytes by acting in a novel autocrine signaling mode to induce the podocytopathy of diabetes and the genesis of albuminuria related with DN. Our findings were in accordance with a previous study which attempted to show that VEGF genetic polymorphisms had a significantly higher frequency in DN patients with microalbuminuria or proteinuria than in DM patients [22]. Since obvious heterogeneity existed, we also performed stratified analysis based on ethnicity and genotyping method. Our results suggested that there was significant correlation of VEGF genetic polymorphisms with an increased risk of DN among Asians and the PCR-RFLP subgroup but not among Caucasians and in the non-PCR-RFLP subgroup, which indicated that ethnic differences and genotype may be the main sources of heterogeneity. All in all, our findings were consistent with previous studies that found VEGF genetic polymorphisms may alter the risk of developing DN in DM patients, suggesting that these polymorphisms may be utilized as biomarkers for early diagnosis and prognosis of $\mathrm{DN}$.

Although this is the first meta-analysis focused on the association of $V E G F$ genetic polymorphisms with the risk of DN in DM patients, our study still has some limitations. Firstly, our results had lacked sufficient statistical power to assess the correlations between genetic variants of VEGF and susceptibility to DN due to the relatively small sample size. Secondly, meta-analysis is a retrospective study that may lead to subject selection bias, thereby affecting the reliability of our results. Thirdly, our meta-analysis failed to obtain original data from the included studies, which may limit further evaluation of potential roles of VEGF genetic polymorphisms in the development and progression of DN. Importantly, the inclusion criteria of cases and controls were not well defined in all included studies, which might also influence our results.
In conclusion, our findings supply convincing evidence that VEGF rs2010963 and rs3025039 polymorphisms may contribute to the risk of DN in DM patients, especially among Asians. Thus, VEGF genetic polymorphisms could be useful biomarkers for early diagnosis of DN in DM patients. However, due to the limitations mentioned above, more studies with larger sample sizes are still needed to provide a more representative statistical analysis accurately.

\section{Conflict of Interests}

The authors have declared that no competing interests exist.

\section{Acknowledgments}

The authors would like to acknowledge the reviewers for their helpful comments on this paper. This work was supported by the Foundation of Social Development Research Program of Liaoning Province (no. 201225094), the National Natural Science Foundation of Liaoning Province (no. 201102252), and the Program for Liaoning Excellent Talents in University.

\section{References}

[1] K. Inoki, H. Mori, J. Wang et al., "mTORC1 activation in podocytes is a critical step in the development of diabetic nephropathy in mice," Journal of Clinical Investigation, vol. 121, no. 6, pp. 2181-2196, 2011.

[2] S. G. Sayyed, A. B. Gaikwad, J. Lichtnekert et al., "Progressive glomerulosclerosis in type 2 diabetes is associated with renal histone $\mathrm{H} 3 \mathrm{~K} 9$ and $\mathrm{H} 3 \mathrm{~K} 23$ acetylation, $\mathrm{H} 3 \mathrm{~K} 4$ dimethylation and phosphorylation at serine 10," Nephrology Dialysis Transplantation, vol. 25, no. 6, pp. 1811-1817, 2010.

[3] A. D. Deshpande, M. Harris-Hayes, and M. Schootman, "Epidemiology of diabetes and diabetes-related complications," Physical Therapy, vol. 88, no. 11, pp. 1254-1264, 2008.

[4] W. Katon, C. R. Lyles, M. M. Parker, A. J. Karter, E. S. Huang, and R. A. Whitmer, "Association of depression with increased risk of dementia in patients with type 2 diabetes: the diabetes 
and aging study," Archives of General Psychiatry, vol. 69, no. 4, pp. 410-417, 2012.

[5] Y. Sakaguchi, T. Shoji, T. Hayashi et al., "Hypomagnesemia in type 2 diabetic nephropathy: a novel predictor of end-stage renal disease," Diabetes Care, vol. 35, no. 7, pp. 1591-1597, 2012.

[6] P. J. Nelson and C. E. Alpers, "Glomerular disease in 2011: new clues to environmental influences in glomerular disease," Nature Reviews Nephrology, vol. 8, no. 2, pp. 65-66, 2012.

[7] L. M. Villeneuve and R. Natarajan, "The role of epigenetics in the pathology of diabetic complications," American Journal of Physiology: Renal Physiology, vol. 299, no. 1, pp. F14-F25, 2010.

[8] Y. S. Kanwar, L. Sun, P. Xie, F. Liu, and S. Chen, "A glimpse of various pathogenetic mechanisms of diabetic nephropathy," Annual Review of Pathology: Mechanisms of Disease, vol. 6, pp. 395-423, 2011.

[9] D. O. Bates, "Vascular endothelial growth factors and vascular permeability," Cardiovascular Research, vol. 87, no. 2, pp. 262271, 2010.

[10] S. Sheikpranbabu, H. Ravinarayanan, B. Elayappan, P. Jongsun, and S. Gurunathan, "Pigment epithelium-derived factor inhibits vascular endothelial growth factor-and interleukinlbeta-induced vascular permeability and angiogenesis in retinal endothelial cells," Vascular Pharmacology, vol. 52, no. 1-2, pp. 84-94, 2010.

[11] A. Dowlati, R. Gray, A. B. Sandler, J. H. Schiller, and D. H. Johnson, "Cell adhesion molecules, vascular endothelial growth factor, and basic fibroblast growth factor in patients with nonsmall cell lung cancer treated with chemotherapy with or without bevacizumab: an eastern cooperative oncology group study," Clinical Cancer Research, vol. 14, no. 5, pp. 1407-1412, 2008.

[12] V. Vincenti, C. Cassano, M. Rocchi, and M. G. Persico, "Assignment of the vascular endothelial growth factor gene to human chromosome 6p21.3," Circulation, vol. 93, no. 8, pp. 1493-1495, 1996.

[13] K. Doi, E. Noiri, and T. Fujita, "Role of vascular endothelial growth factor in kidney disease," Current Vascular Pharmacology, vol. 8, no. 1, pp. 122-128, 2010.

[14] J. W. Miller, J. Le Couter, E. C. Strauss, and N. Ferrara, "Vascular endothelial growth factor a in intraocular vascular disease," Ophthalmology, vol. 120, no. 1, pp. 106-114, 2013.

[15] K. Kaul, A. Hodgkinson, J. M. Tarr, E. M. Kohner, and R. Chibber, "Is inflammation a common retinal-renal-nerve pathogenic link in diabetes?" Current Diabetes Reviews, vol. 6, no. 5, pp. 294-303, 2010.

[16] J. Long, Y. Wang, W. Wang, B. H. J. Chang, and F. R. Danesh, "Identification of microRNA-93 as a novel regulator of vascular endothelial growth factor in hyperglycemic conditions," The Journal of Biological Chemistry, vol. 285, no. 30, pp. 2345723465, 2010.

[17] M. Mironidou-Tzouveleki, S. Tsartsalis, and C. Tomos, "Vascular endothelial growth factor (VEGF) in the pathogenesis of diabetic nephropathy of type 1 diabetes mellitus," Current Drug Targets, vol. 12, no. 1, pp. 107-114, 2011.

[18] A. Bonnefond, P. Saulnier, M. G. Stathopoulou et al., "What is the contribution of two genetic variants regulating VEGF levels to type 2 diabetes risk and to microvascular complications?" PLoS ONE, vol. 8, no. 2, Article ID e55921, 2013.

[19] A. Avogaro, M. Albiero, L. Menegazzo, S. de Kreutzenberg, and G. P. Fadini, "Endothelial dysfunction in diabetes: the role of reparatory mechanisms," Diabetes Care, vol. 34, supplement 2, pp. S285-S290, 2011.
[20] F. C. Brosius III, C. C. Khoury, C. L. Buller, and S. Chen, "Abnormalities in signaling pathways in diabetic nephropathy," Expert Review of Endocrinology and Metabolism, vol. 5, no. 1, pp. 51-64, 2010.

[21] J. Tavakkoly-Bazzaz, M. M. Amoli, V. Pravica et al., "VEGF gene polymorphism association with diabetic neuropathy," Molecular Biology Reports, vol. 37, no. 7, pp. 3625-3630, 2010.

[22] A. McKnight, A. P. Maxwell, C. C. Patterson, H. R. Brady, and D. A. Savage, "Association of VEGF-1499C $\rightarrow$ T polymorphism with diabetic nephropathy in type 1 diabetes mellitus," Journal of Diabetes and its Complications, vol. 21, no. 4, pp. 242-245, 2007.

[23] A. Nikzamir, A. Esteghamati, A. A. Hammedian, and T. Mahmoudi, "The role of vascular endothelial growth factor +405 G/C polymorphism and albuminuria in patients with type 2 diabetes mellitus," Molecular Biology Reports, vol. 39, no. 2, pp. 881-886, 2012.

[24] M. Buraczynska, P. Ksiazek, I. Baranowicz-Gaszczyk, and L. Jozwiak, "Association of the VEGF gene polymorphism with diabetic retinopathy in type 2 diabetes patients," Nephrology Dialysis Transplantation, vol. 22, no. 3, pp. 827-832, 2007.

[25] Q. Xiao, Y. Zhang, N. Zhang, C. H. Yu, and X. H. Fu, "Correlation of vascular endothelial growth factor gene C2587A polymorphism to diabetic nephropathy in type 2 diabetes mellitus," Journal of Hunan Normal University (Medical Science), vol. 8, no. 1, pp. 24-26, 2011.

[26] X. H. Zhang, Y. Guo, L. H. Chen et al., "C936T polymorphism in $3^{\prime}$-untranslated region of vascular endothelial growth factor gene is associated with diabetic nephropathy in type 2 diabetics," Chinese Journal of Endocrinology and Metabolism, vol. 24, no. 3, pp. 299-301, 2008.

[27] A. Stang, "Critical evaluation of the Newcastle-Ottawa scale for the assessment of the quality of nonrandomized studies in metaanalyses," European Journal of Epidemiology, vol. 25, no. 9, pp. 603-605, 2010.

[28] E. Zintzaras and J. P. A. Ioannidis, "HEGESMA: genome search meta-analysis and heterogeneity testing," Bioinformatics, vol. 21, no. 18, pp. 3672-3673, 2005.

[29] J. L. Peters, A. J. Sutton, D. R. Jones, K. R. Abrams, and L. Rushton, "Comparison of two methods to detect publication bias in meta-analysis," The Journal of the American Medical Association, vol. 295, no. 6, pp. 676-680, 2006.

[30] B. Q. Guo, Y. G. Cao, Q. J. Zhang et al., "Association of the vascular endothelial growth factor gene polymorphism to diabetic nephropathy in type 2 diabetes mellitus," Chinese Journal of Diabetes, vol. 16, pp. 465-468, 2008.

[31] H. He, C. J. Jin, X. J. He et al., "Association of the vascular endothelial growth factor gene polymorphism to diabetic nephropathy in type 2 diabetes mellitus," Journal of Practical Diabetology, vol. 3, pp. 25-27, 2007.

[32] R. F. Li, Y. N. Wang, and Y. Li, "Correlation of vascular endothelial growth factor gene polymorphism to diabetic nephropathy in type 2 diabetic mellitus," Chinese Journal of Nephrology, vol. 21, pp. 28-31, 2005.

[33] F. Xue, H. L. Liu, Y. N. Wang et al., "Relationship of the level and gene polymorphism of vascular endothelial growth factor with type 2 diabetic nephropathy," Chinese Journal of Nephrology, vol. 23, pp. 813-814, 2007.

[34] D. D. Zhang, G. Y. Wang, and X. X. Wang, "Association between polymorphism of C936T in vascular endothelial growth factor gene and type 2 diabetic nephropathy," Journal of Practical Medicine, vol. 24, pp. 1904-1906, 2008. 
[35] M. Shibuya, "Vascular endothelial growth factor and its receptor system: physiological functions in angiogenesis and pathological roles in various diseases," Journal of Biochemistry, vol. 153, no. 1, pp. 13-19, 2013.

[36] G. Pavlisa, V. Kusec, S. O. Kolonic, A. S. Markovic, and B. Jaksic, "Serum levels of VEGF and bFGF in hypoxic patients with exacerbated COPD," European Cytokine Network, vol. 21, no. 2, pp. 92-98, 2010.

[37] R. O. Schlingemann, C. J. F. Van Noorden, M. J. M. Diekman et al., "VEGF levels in plasma in relation to platelet activation, glycemic control, and microvascular complications in type 1 diabetes," Diabetes Care, vol. 36, no. 6, pp. 1629-1634, 2013.

[38] D. R. Senger, "Vascular endothelial growth factor: much more than an angiogenesis factor," Molecular Biology of the Cell, vol. 21, no. 3, pp. 377-379, 2010.

[39] S. Chen and F. N. Ziyadeh, "Vascular endothelial growth factor and diabetic nephropathy," Current Diabetes Reports, vol. 8, no. 6, pp. 470-476, 2008.

[40] T. Nakagawa, T. Kosugi, M. Haneda, C. J. Rivard, and D. A. Long, "Abnormal angiogenesis in diabetic nephropathy," Diabetes, vol. 58, no. 7, pp. 1471-1478, 2009.

[41] X. Yang, Y. Deng, H. Gu et al., "Polymorphisms in the vascular endothelial growth factor gene and the risk of diabetic retinopathy in Chinese patients with type 2 diabetes," Molecular Vision, vol. 17, pp. 3088-3096, 2011. 